\title{
An Enhanced Preventive Maintenance Optimization Model Based on a Three-Stage Failure Process
}

\author{
Ruifeng Yang, ${ }^{1}$ Jianshe Kang, ${ }^{1}$ and Zhenya Quan ${ }^{2}$ \\ ${ }^{1}$ Mechanical Engineering College, No. 97, Heping West Road, Shijiazhuang, Hebei 050000, China \\ ${ }^{2}$ Shanxi Conservancy Technical College, Taiyuan, Shanxi 030000, China \\ Correspondence should be addressed to Ruifeng Yang; rfyangphm@163.com
}

Received 14 January 2015; Revised 1 March 2015; Accepted 16 March 2015

Academic Editor: Francesco Di Maio

Copyright (c) 2015 Ruifeng Yang et al. This is an open access article distributed under the Creative Commons Attribution License, which permits unrestricted use, distribution, and reproduction in any medium, provided the original work is properly cited.

\begin{abstract}
Nuclear power plants are highly complex systems and the issues related to their safety are of primary importance. Probabilistic safety assessment is regarded as the most widespread methodology for studying the safety of nuclear power plants. As maintenance is one of the most important factors for affecting the reliability and safety, an enhanced preventive maintenance optimization model based on a three-stage failure process is proposed. Preventive maintenance is still a dominant maintenance policy due to its easy implementation. In order to correspond to the three-color scheme commonly used in practice, the lifetime of system before failure is divided into three stages, namely, normal, minor defective, and severe defective stages. When the minor defective stage is identified, two measures are considered for comparison: one is that halving the inspection interval only when the minor defective stage is identified at the first time; the other one is that if only identifying the minor defective stage, the subsequent inspection interval is halved. Maintenance is implemented immediately once the severe defective stage is identified. Minimizing the expected cost per unit time is our objective function to optimize the inspection interval. Finally, a numerical example is presented to illustrate the effectiveness of the proposed models.
\end{abstract}

\section{Introduction}

It is well recognized that nuclear power plants (NPPs) are tightly regulated complex systems, where the issues related to their safety are of primary importance [1]. A possible and widely used way of analyzing the performance of safety for NPPs is to perform probabilistic safety assessments (PSA). PSA constitutes the most widespread methodology for studying the safety of NPPs and plays an increasing role in safety analysis and in developing safety improvements for industrial systems [2]. One of the important tasks of PSA for NPPs is evaluating the system reliability in terms of quantifiable measures. It is well known that there are many factors that affect the total reliability of NPPs. Among them, maintenance has been considered as one of the most important factors $[3,4]$.

The purpose of maintenance in nuclear applications is to identify and militate the degradation of plant or system, as well as to restore the functions of system to an acceptable level, thus improving the reliability of NPPs. The optimization of maintenance programs could be summarized as "the right work, on the right equipment, at the right time" [5]. Here the "right time" refers to scientific and appropriate decisionmaking for initiating a maintenance program [6].

In this paper, our aim is to develop maintenance models to optimize the interval of maintenance programs in terms of "right time." Numerous maintenance policies have been implemented on the NPPs to prevent the occurrence of failure [7]. Preventive maintenance (PM) is perhaps one of the most popular maintenance policies, by which maintenance activities are executed with a planned interval aiming at preventing potential failures from occurring $[8,9]$. PM is still a dominant maintenance policy due to its easy implementation in industrial applications.

As we all know, inspection as an important PM activity could reveal the status of the system being inspected; thus it helps maintenance engineers make decisions to avoid the occurrence of failure [10]. Inspections may be performed discretely with a periodic or aperiodic interval by using inspection instrument or continuously by modern condition 
monitoring devices. The periodic inspection activities are common in industry because of the effectiveness and convenience.

However, how often do engineers inspect the plants or the determination of inspection interval is still a key issue. The traditional determination of inspection interval is decided by managers' experience. Of course it is not scientific. Many researchers have developed numerous PM optimization models to search the optimal inspection interval under various modeling conditions [11-13]. But the models based on the delay time concept have been proved to have the obvious advantages for optimizing the inspection interval since the delay-time technique can directly model the relationship between the inspection intervention and the system performance in contrast with other PM models [14, 15].

The delay time concept was first introduced by Christer in 1976 [16], which defines the failure process as a two-stage failure process, namely, normal stage and delay time stage. The normal stage is from new to an initial point that a defect can be first identified by an inspection and the delay time stage from this initial point to failure. By the definition of delay time concept, the plant can be in one of two states before failure, namely, normal and defective. A defect can be identified if the inspection is carried out during the delay time stage. Many inspection and PM models, especially successful case studies based on the two-stage failure process, have been reported with actual applications in industry [17].

However, in industrial applications, the systems may be described more than two states before failure as the three-color scheme is mostly used to divide the state before failure into green (normal), yellow (need attention), and red (need immediate attention) [18]. Considering this industrial scenario, Wang [19] firstly extended the two-stage failure process into a three-stage failure process, which is closer to the practical situation and provides more decision options for different states. In the work [19], the inspection interval is shortened to be half of the current interval to more frequently inspect the system when the minor defective stage is identified but immediately replace the system if it is in the severe defective stage. Wang et al. [20] further considered a two-level inspection policy with PM and delaying the maintenance once the severe defective stage is identified and the time interval to the next PM is less than a threshold level. Yang et al. [21, 22] proposed an inspection maintenance optimization model with imperfect maintenance based on a three-stage failure process. An inspection optimization model based on a three-stage failure process is presented by Yang et al. [23].

The contribution that Wang extended the failure process into three stages is meaningful, as this extension is closer to reality and corresponds to the industrial scenario. When the minor defective stage is identified, halving the inspection interval is adopted. However, one may argue that how often do engineers halve the inspection interval. It is done only when the minor defective stage is identified at the first time or if only identifying the minor defective stage because the former may be easily implemented for maintenance operators but the latter can inspect the condition of system frequently and avoid the failure to some extent. This question is not explained in the previous work based on the three-stage failure process.

Therefore, in this paper we propose an enhanced preventive maintenance optimization model based on a threestage failure process to improve the reliability of systems, thus laying the foundation for safety assessment of NPPs. The lifetime of system before failure is divided into three stages, namely, normal, minor defective, and severe defective stages. When system fails, replacement is carried out. Once the severe defective stage is identified, the maintenance is implemented immediately. But when the minor defective stage is identified, we adopt two measures for comparison: one is that halving the inspection interval only when the minor defective stage is identified at the first time; the other one is that if only identifying the minor defective stage, the subsequent inspection interval is halved. Minimizing the expected cost per unit time is our objective function to optimize the inspection interval.

The contributions of this paper are as follows: (1) an enhanced preventive maintenance optimization model based on the three-stage failure process is presented to lay the foundation for safety assessment of NPPs; (2) the deterioration of system is subject to the three-stage failure process; (3) two different maintenance decisions are considered for comparison when the minor defective stage is identified.

The remaining parts of the paper are organized as follows: Section 2 presents the modeling assumptions and notation. Section 3 provides the proposed cost models with perfect inspection case and imperfect inspection case is modeled in Section 4. Section 5 gives numerical examples. Finally, Section 6 concludes the paper and the future researches are suggested.

\section{Modeling Assumptions and Notation}

The following assumptions and notation are presented for the subsequent modeling.

(1) The failure process of single component system is divided into three stages: normal stage $U$, minor defective stage $V$, and severe defective stage $H$. These three stages are assumed to be independent.

(2) The system is inspected periodically with an interval $t$.

(3) Both perfect and imperfect inspection cases are considered. Perfect inspection can always reveal the defective stage no matter it is minor and severe. However, imperfect inspection may miss the minor defective stage with a probability of $(1-r)$ but always can identify the severe defective stage.

(4) If the system is found in the normal stage, do nothing. Failure can be observed immediately and replacement is always carried out at once.

(5) Once system is found to be in the severe defective stage, it is always repaired immediately.

(6) As to the minor defective stage, two actions are considered here: the first one is to shorten the inspection interval only when the minor defective stage is 
identified at the first time; the other one is to shorten the inspection interval if only the minor defective stage is identified.

(7) Repair or replacement is regarded as renewing the system, though it may be the only option for a single component system.

The subsequent modeling will use the following notations:

$U, f_{U}(u)$, and $F_{U}(u)$ : random variable representing the duration of normal stage, probability density function (pdf), and cumulative distribution function (pdf) of normal stage;

$V, f_{V}(v)$, and $F_{V}(v)$ : random variable representing the duration of minor defective stage, pdf, and cdf of minor defective stage;

$H, f_{H}(h)$, and $F_{H}(h)$ : random variable representing the duration of severe defective stage, pdf, and cdf of severe defective stage;

$t$ : inspection interval;

$T_{f}$ : random failure time;

$T_{p}$ : random time of an inspection renewal due to the severe defective stage identification by an inspection;

$r$ : identification probability of the minor defective stage with imperfect inspection;

$C_{s}$ : average cost per inspection;

$C_{f}$ : average cost per failure;

$C_{p}$ : average cost caused by an inspection renewal due to identification of the severe defective stage;

$E C(t)$ : expected renewal cycle cost;

$E C_{f}(t)$ : expected cost caused by failure renewal;

$E C_{s}(t)$ : expected cost caused by inspection renewal due to identification of severe defective stage;

$E L(t)$ : expected renewal cycle length;

$E L_{f}(t)$ : expected length caused by failure renewal;

$E L_{s}(t)$ : expected length caused by inspection renewal due to identification of severe defective stage;

$C(t)$ : expected cost per unit time.

\section{Cost Models with Perfect Inspection}

Two cost models are formulated in this section based on the different decisions when identifying the minor defective stage. Perfect inspection means that inspections can identify the defective stages no matter the defect is minor or severe. The objective function of the models is to minimize the expected cost per unit time for deriving the optimal inspection interval. There are two renewal scenarios, namely, a failure renewal and an inspection renewal caused by identifying the severe defective stage. According to the renewal

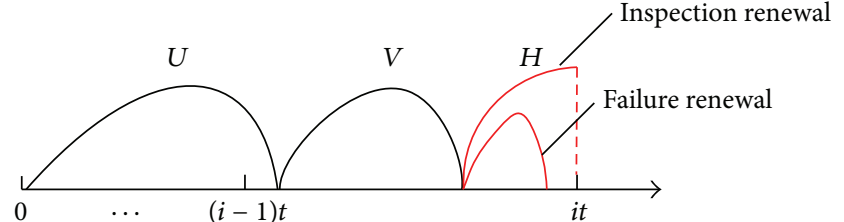

FIGURE 1: The system is renewed before the minor defective stage is identified.

theory, the renewal probabilities need to be derived before formulating these cost models.

Model 1. The inspection interval is shortened only when the minor defective stage is identified at the first time.

3.1. The System May Be Renewed before the Minor Defective Stage Is Identified. There could exist two renewal scenarios, as shown in Figure 1.

(1) A failure renewal happens when the system deteriorates into the minor defective stage and then fails in $((i-1) t$, it $)$ simultaneously. As no minor defective stage is identified, the total length of minor defective stage and severe defective stage is shorter than the inspection interval $t$. The probability of such a failure renewal is

$$
\begin{aligned}
& P\left((i-1) t<T_{f}<i t\right) \\
& =P((i-1) t<U<i t, 0<V<i t-U, \\
& \quad 0<H<i t-U-V) \\
& =\int_{(i-1) t}^{i t} f_{U}(u) \int_{0}^{i t-u} f_{V}(v) F_{H}(i t-u-v) d v d u .
\end{aligned}
$$

(2) An inspection renewal occurs once the severe defective stage is found at it by an inspection. The minor defective stage is within $((i-1) t, i t)$ and severe defective stages are longer than $i t-u-v$, where $u$ and $v$ are the duration of the normal and minor defective stages. The probability of such an inspection renewal is

$$
\begin{aligned}
& P\left(T_{p}=i t\right) \\
& \quad=P((i-1) t<U<i t, 0<V<i t-U, \\
& H>i t-U-V) \\
& \quad=\int_{(i-1) t}^{i t} f_{U}(u) \int_{0}^{i t-u} f_{V}(v)\left(1-F_{H}(i t-u-v)\right) d v d u .
\end{aligned}
$$

According to renewal probabilities, the expected renewal cycle cost and length with different renewal scenarios can be derived. 
3.1.1. Expected Renewal Cycle Cost $E C_{1}(t)$. If the system fails in $((i-1) t$, it $)$ and failure renewal happens, the relevant cost is $\left(C_{f}+(i-1) C_{s}\right)$, including the cost of $(i-1)$ th inspection and failure replacement cost. But once the severe defective stage is identified at it and inspection renewal happens, the relevant cost is $\left(C_{p}+i C_{s}\right)$, including the cost of $i$ th inspection and maintenance cost. The relevant cost of each event multiplies by the renewal probability, respectively; thus the expected renewal cycle cost $E C_{1}(t)$ can be derived as

$$
\begin{aligned}
& E C_{1}(t) \\
& \begin{aligned}
=\sum_{i=1}^{\infty}( & \left(C_{f}+(i-1) C_{s}\right) P\left((i-1) t<T_{f}<i t\right) \\
& \left.+\left(C_{p}+i C_{s}\right) P\left(T_{p}=i t\right)\right) \\
=\sum_{i=1}^{\infty}( & \left(C_{f}+(i-1) C_{s}\right) \\
& \cdot \int_{(i-1) t}^{i t} f_{U}(u) \int_{0}^{i t-u} f_{V}(v) F_{H}(i t-u-v) d v d u \\
& +\left(C_{p}+i C_{s}\right) \\
& \cdot \int_{(i-1) t}^{i t} f_{U}(u) \\
& \left.\cdot \int_{0}^{i t-u} f_{V}(v)\left(1-F_{H}(i t-u-v)\right) d v d u\right) .
\end{aligned}
\end{aligned}
$$

3.1.2. Expected Renewal Cycle Length $E L_{1}(t)$. Before deriving the expected cycle length of failure renewal, the pdf of system fails at $T_{f}, T_{f} \in P\left((i-1) t+h<T_{f}<(i-1) t+h+d h\right)$ should be formulated firstly using the same way as (1)

$$
\begin{aligned}
P( & \left.(i-1) t+h<T_{f}<(i-1) t+h+d h\right) \\
= & \int_{(i-1) t}^{(i-1) t+h} f_{U}(u) \\
& \quad \cdot \int_{0}^{(i-1) t+h-u} f_{V}(v) f_{H}((i-1) t+h-u-v) d v d u d h .
\end{aligned}
$$

The expected renewal cycle length can be derived as the way as (3). The relevant length of each event multiplies by the renewal probability, respectively; thus the expected renewal cycle length $E L_{1}(t)$ can be derived as

$$
\begin{aligned}
& E L_{1}(t) \\
& \qquad \sum_{i=1}^{\infty}\left(\int_{0}^{t}((i-1) t+h)\right. \\
& \cdot P\left((i-1) t+h<T_{f}<(i-1) t+h+d h\right) \\
& \left.+i t P\left(T_{p}=i t\right)\right)
\end{aligned}
$$

$$
\begin{aligned}
& =\sum_{i=1}^{\infty}\left(\int_{0}^{t}((i-1) t+h)\right. \\
& \quad \cdot \int_{(i-1) t}^{(i-1) t+h} f_{U}(u) \\
& \quad \cdot \int_{0}^{(i-1) t+h-u} f_{V}(v) f_{H} \\
& \quad \cdot((i-1) t+h-u-v) d v d u d h \\
& +i t \int_{(i-1) t}^{i t} f_{U}(u) \\
& \left.\cdot \int_{0}^{i t-u} f_{V}(v)\left(1-F_{H}(i t-u-v)\right) d v d u\right) .
\end{aligned}
$$

3.2. The System Is Renewed after the Minor Defective Stage Is Identified at it. There also exist two renewal scenarios, as shown in Figure 2.

(1) The system fails in $(i t+(n-1) t / 2$, it $+n t / 2)$ after the minor defective stage is identified at $i t$. The minor defective stage starts within $((i-1) t$, it) and ends within $(i t+(n-1) t / 2$, it $+n t / 2)$. The severe defective stage and failure happens in the same interval. The probability of such a renewal is

$$
\begin{gathered}
P\left(i t+\frac{(n-1) t}{2}<T_{f}<i t+\frac{n t}{2}\right) \\
=P\left((i-1) t<U<i t, i t+\frac{(n-1) t}{2}\right. \\
-U<V<i t+\frac{n t}{2}-U, 0<H<i t \\
\left.\quad+\frac{n t}{2}-U-V\right) \\
=\int_{(i-1) t}^{i t} f_{U}(u) \int_{i t+(n-1) t / 2-u}^{i t+n t / 2-u} f_{V}(v) F_{H} \\
\cdot\left(i t+\frac{n t}{2}-u-v\right) d v d u .
\end{gathered}
$$

(2) The system is renewed at it $+n t / 2$ as the severe defective stage is firstly identified. The normal stage ends within $((i-1) t, i t)$. The minor defective stage is firstly identified at it and ends within $(i t+(n-1) t / 2$, $i t+n t / 2)$. So the severe defective stage is longer than $i t+n t / 2-u-v$. The probability of such a renewal is

$$
\begin{gathered}
P\left(T_{p}=i t+\frac{n t}{2}\right) \\
=P\left((i-1) t<U<i t, i t+\frac{(n-1) t}{2}\right. \\
-U<V<i t+\frac{n t}{2}-U, H>i t \\
\left.+\frac{n t}{2}-U-V\right)
\end{gathered}
$$




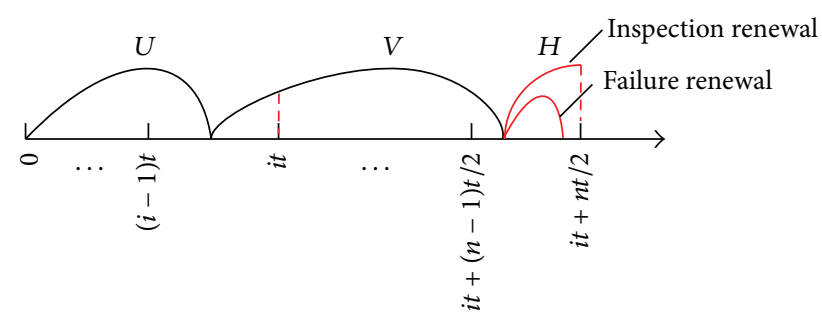

FIGURE 2: The system is renewed after the minor defective stage is identified at the first time.

$$
\begin{aligned}
=\int_{(i-1) t}^{i t} f_{U}(u) \int_{i t+(n-1) t / 2-u}^{i t+n t / 2-u} f_{V}(v) \\
\\
\cdot\left(1-F_{H}\left(i t+\frac{n t}{2}-u-v\right)\right) d v d u
\end{aligned}
$$

Therefore, the expected renewal cycle cost and length under different renewal scenarios can be given as follows.

3.2.1. Expected Renewal Cycle Cost $E C_{2}(t)$. When the system fails in $(i t+(n-1) t / 2$, it $+n t / 2)$, the relevant cost is $\left(C_{f}+(i+\right.$ $\left.n-1) C_{s}\right)$, including the cost of $(i+n-1)$ th inspection and failure replacement cost. But once the severe defective stage is identified at it $+n t / 2$, the relevant cost is $\left(C_{p}+(i+n) C_{s}\right)$, including the cost of $(i+n)$ th inspection and maintenance cost. The relevant cost of each renewal event multiplies by the renewal probability, respectively; thus the expected renewal cycle cost $E C_{2}(t)$ can be derived as

$$
\begin{aligned}
& E C_{2}(t) \\
& =\sum_{i=1}^{\infty} \sum_{n=1}^{\infty}\left(\left(C_{f}+(i+n-1) C_{s}\right)\right. \\
& \cdot P\left(i t+\frac{(n-1) t}{2}<T_{f}<i t+\frac{n t}{2}\right) \\
& \left.+\left(C_{p}+(i+n) C_{s}\right) P\left(T_{p}=i t+\frac{n t}{2}\right)\right) \\
& =\sum_{i=1}^{\infty} \sum_{n=1}^{\infty}\left(C_{f}+(i+n-1) C_{s}\right) \\
& \cdot \int_{(i-1) t}^{i t} f_{U}(u) \int_{i t+(n-1) t / 2-u}^{i t+n t / 2-u} f_{V}(v) F_{H} \\
& +\left(C_{p}+(i+n) C_{s}\right) \\
& +\int_{(i-1) t}^{i t} f_{U}(u) \\
& +\left(i t+\frac{n t}{2}-u-v\right) d v d u
\end{aligned}
$$

$$
\begin{aligned}
& \cdot \int_{i t+(n-1) t / 2-u}^{i t+n t / 2-u} f_{V}(v) \\
& \left.\cdot\left(1-F_{H}\left(i t+\frac{n t}{2}-u-v\right)\right) d v d u\right) .
\end{aligned}
$$

3.2.2. Expected Renewal Cycle Length $E L_{2}(t)$. The relevant length of each event multiplies by the renewal probability, respectively; thus the expected renewal cycle length $E L_{2}(t)$ can be derived as

$$
\begin{aligned}
& E L_{2}(t) \\
& =\sum_{i=1}^{\infty} \sum_{n=1}^{\infty}\left(\int_{0}^{t / 2}\left(i t+\frac{(n-1) t}{2}+h\right)\right. \\
& \cdot \int_{(i-1) t}^{i t} f_{U}(u) \\
& \cdot \int_{i t+(n-1) t / 2-u}^{i t+(n-1) t / 2+h-u} f_{V}(v) f_{H} \\
& \cdot\left(i t+\frac{(n-1) t}{2}+h-u-v\right) d v d u d h \\
& +\left(i t+\frac{n t}{2}\right) \\
& \cdot \int_{(i-1) t}^{i t} f_{U}(u) \\
& \cdot \int_{i t+(n-1) t / 2-u}^{i t+n t / 2-u} f_{V}(v) \\
& \left.\cdot\left(1-F_{H}\left(i t+\frac{n t}{2}-u-v\right)\right) d v d u\right) .
\end{aligned}
$$

3.2.3. Expected Cost per Unit Time $C(t)$. Based on the expected cycle cost and length, expected cost per unit time can be calculated using the renewed reward theory [24] as

$$
C(t)=\frac{E C(t)}{E L(t)}=\frac{E C_{1}(t)+E C_{2}(t)}{E L_{1}(t)+E L_{2}(t)} .
$$

Model 2. The inspection interval is shortened if only the minor defective stage is identified.

\subsection{The System Is Renewed before Founding the Minor Defec- tive Stage. This scenario is the same as Section 3.1.}

3.4. The System Is Renewed after Identifying the Minor Defective Stage. As this model supposes that if only the minor defective stage is identified, the subsequent inspection interval is halved. Here, we consider the scenario that system is renewed after the minor defective stage is identified at $k$ th time and the inspection interval is shortened to be $t / 2^{k}$ for generality. 


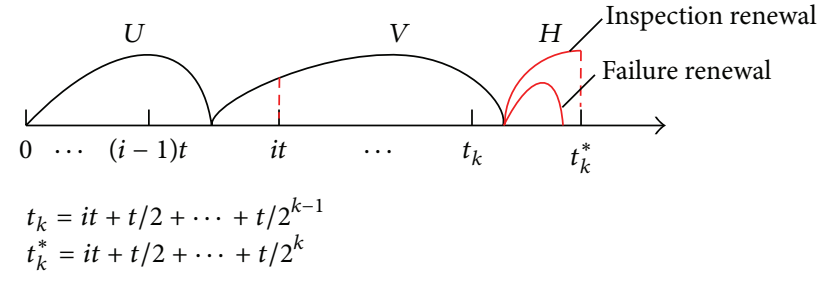

FIgURE 3: The system is renewed after the minor defective stage is identified at $k$ th time.

The minor defective stage starts within $((i-1) t, i t)$ and is found at it by an inspection; then the interval is halved. However, the subsequent inspections also identify the minor defective stage; the inspection interval is accordingly halved. The system is renewed until the minor defective stage is identified at $k$ th time. There also include two different scenarios, as depicted in Figure 3.

(1) The system is renewed when fails in $\left(t_{k}, t_{k}^{*}\right)$. The system deteriorates into the minor defective stage within $((i-1) t, i t)$ and the minor defect is firstly identified at $i t$ by an inspection. So the subsequent inspection interval is halved. Finally, the minor defective stage ends within $\left(t_{k}, t_{k}^{*}\right)$ and the severe defective stage and failure happens in the same interval. The probability of such a failure renewal is

$$
\begin{aligned}
& P\left(t_{k}<T_{f}<t_{k}^{*}\right) \\
& =P\left((i-1) t<U<i t, t_{k}-U<V<t_{k}^{*}-U,\right. \\
& \left.\quad 0<H<t_{k}^{*}-U-V\right) \\
& =\int_{(i-1) t}^{i t} f_{U}(u) \int_{t_{k}-u}^{t_{k}^{*}-u} f_{V}(v) F_{H}\left(t_{k}^{*}-u-v\right) d v d u .
\end{aligned}
$$

(2) The system is replaced when the inspection renewal happened. The minor defective stage is firstly identified at it and ends within $\left(t_{k}, t_{k}^{*}\right)$. The severe defective stage is longer than $t_{k}^{*}-u-v$ and identified at $t_{k}^{*}$, leading to the system renewal. The probability of this inspection renewal is

$$
\begin{aligned}
& P\left(T_{p}=t_{k}^{*}\right) \\
& =P\left((i-1) t<U<i t, t_{k}-U<V<t_{k}^{*}-U,\right. \\
& \left.H>t_{k}^{*}-U-V\right) \\
& =\int_{(i-1) t}^{i t} f_{U}(u) \int_{t_{k}-u}^{t_{k}^{*}-u} f_{V}(v)\left(1-F_{H}\left(t_{k}^{*}-u-v\right)\right) d v d u .
\end{aligned}
$$

Accordingly, the expected renewal cycle cost and length under different renewal scenarios can be given using the same way as model 1.
3.4.1. Expected Renewal Cycle Cost $E C_{3}(t)$. When the system fails in $\left(t_{k}, t_{k}^{*}\right)$, the relevant cost is $\left(C_{f}+(i+k-1) C_{s}\right)$, including the cost of $(i+k-1)$ th inspection and failure replacement cost. But if the severe defective stage is identified at $t_{k}^{*}$, the relevant cost is $\left(C_{p}+(i+k) C_{s}\right)$, including the cost of $(i+k)$ th inspection and maintenance cost. The relevant cost of each event multiplies by the renewal probability, respectively; thus the expected renewal cycle cost $E C_{3}(t)$ can be derived as

$$
\begin{aligned}
& E C_{3}(t) \\
& =\sum_{i=1}^{\infty}\left(\left(C_{f}+(i+k-1) C_{s}\right) P\left(t_{k}<T_{f}<t_{k}^{*}\right)\right. \\
& \left.+\left(C_{p}+(i+k) C_{s}\right) P\left(T_{p}=t_{k}^{*}\right)\right) \\
& =\sum_{i=1}^{\infty}\left(\left(C_{f}+(i+k-1) C_{s}\right)\right. \\
& \cdot \int_{(i-1) t}^{i t} f_{U}(u) \\
& \cdot \int_{t_{k}-u}^{t_{k}^{*}-u} f_{V}(v) F_{H}\left(t_{k}^{*}-u-v\right) d v d u \\
& +\left(C_{p}+(i+k) C_{s}\right) \\
& \cdot \int_{(i-1) t}^{i t} f_{U}(u) \\
& \left.\cdot \int_{t_{k}-u}^{t_{k}^{*}-u} f_{V}(v)\left(1-F_{H}\left(t_{k}^{*}-u-v\right)\right) d v d u\right) .
\end{aligned}
$$

3.4.2. Expected Renewal Cycle Length $E L_{3}(t)$. The relevant length of each event multiplies by the renewal probability, respectively; thus the expected renewal cycle length $E L_{3}(t)$ can be derived as

$$
\begin{aligned}
& E L_{3}(t) \\
& \qquad \sum_{i=1}^{\infty}\left(\int_{0}^{t / 2^{k}}\left(t_{k}+h\right) \int_{(i-1) t}^{i t} f_{U}(u)\right. \\
& \cdot \int_{t_{k}-u}^{t_{k}+h-u} f_{V}(v) f_{H} \\
& \cdot\left(t_{k}+h-u-v\right) d v d u d h \\
& +t_{k}^{*} \int_{(i-1) t}^{i t} f_{U}(u) \\
& \cdot \int_{t_{k}-u}^{t_{k}^{*}-u} f_{V}(v) \\
& \left.\cdot\left(1-F_{H}\left(t_{k}^{*}-u-v\right)\right) d v d u\right) .
\end{aligned}
$$


3.4.3. Expected Cost per Unit Time $C(t)$. Based on the derived expected cycle cost and length, expected cost per unit time can be calculated using the same way as (10)

$$
C(t)=\frac{E C(t)}{E L(t)}=\frac{E C_{1}(t)+E C_{3}(t)}{E L_{1}(t)+E L_{3}(t)} .
$$

\section{Cost Models with Imperfect Inspection}

Imperfect inspection means that inspections may not identify the minor defective stage perfectly but can identify the severe defective stage perfectly. Two different cost models with imperfect inspection are given in this section to optimize the inspection intervals. Similarly, two renewal scenarios are considered here and the renewal probabilities should be formulated. According to the different decisions when identifying the minor defective stage, two optimization models are proposed.

Model 1. The inspection interval is shortened only when the minor defective stage is identified at the first time.

4.1. The System Is Renewed before the Minor Defective Stage Is Identified, as Shown in Figure 4. There may exist two renewal scenarios.

(1) The system is renewed when it fails in $((i-1) t$, it). The normal stage ends and then the minor defective stage starts within $((j-1) t, j t)$. The length of minor defective stage is longer than $((i-1) t-u)$ but all the inspections in $(j t,(i-1) t)$ miss the minor defect because of the imperfect inspection. The probability is given as

$$
\begin{gathered}
P\left((i-1) t<T_{f}<i t\right) \\
=P((j-1) t<U<j t,(i-1) t-U<V<i t-U, \\
0<H<i t-U-V) \\
=\sum_{j=1}^{i} \int_{(j-1) t}^{j t} \int_{\delta((i-1) t-u)}^{i t-u}(1-r)^{i-j} f_{U}(u) \\
\cdot f_{V}(v) F_{H}(i t-u-v) d v d u,
\end{gathered}
$$

where we define $\delta(u)=\max (u, 0)$.

(2) The system is renewed at it as the severe defective stage is identified by an inspection. The minor defective stage starts within $((j-1) t, j t)$ and ends within $((i-1) t, i t)$. Of course, all the inspections within $(j t,(i-1) t)$ miss the minor defective stage. The probability is

$$
\begin{aligned}
& P\left(T_{p}=i t\right) \\
& =P((j-1) t<U<j t,(i-1) t-U<V<i t-U, \\
& \quad H>i t-U-V)
\end{aligned}
$$

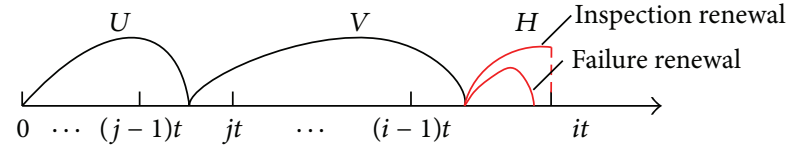

FIGURE 4: The system is renewed before minor defective stage is identified with imperfect inspection.

$$
\begin{gathered}
=\sum_{j=1}^{i} \int_{(j-1) t}^{j t} \int_{\delta((i-1) t-u)}^{i t-u}(1-r)^{i-j} f_{U}(u) f_{V}(v) \\
\cdot\left(1-F_{H}(i t-u-v)\right) d v d u .
\end{gathered}
$$

Consequently, the expected renewal cycle cost and length with different renewal scenarios can be derived.

4.1.1. Expected Renewal Cycle Cost $E C_{11}(t)$. When the system fails in $((i-1) t, i t)$, the relevant maintenance cost is $\left((i-1) C_{s}+\right.$ $\left.C_{f}\right)$. However, once the severe defective stage is identified at it by an inspection, the relevant cost is $\left(i C_{s}+C_{p}\right)$. Therefore, the expected renewal cycle cost $E C_{11}(t)$ can be derived through multiplying the relevant cost of each event by the renewal probability, respectively;

$$
\begin{gathered}
E C_{11}(t) \\
=\sum_{i=1}^{\infty}\left(\left(C_{f}+(i-1) C_{s}\right) P\left((i-1) t<T_{f}<i t\right)\right. \\
\left.+\left(C_{p}+i C_{s}\right) P\left(T_{p}=i t\right)\right) \\
=\sum_{i=1}^{\infty}\left(\left(C_{f}+(i-1) C_{s}\right)\right. \\
\cdot \sum_{j=1}^{i} \int_{(j-1) t}^{j t} \int_{\delta((i-1) t-u)}^{i t-u}(1-r)^{i-j} \\
\quad \cdot f_{U}(u) f_{V}(v) F_{H}(i t-u-v) d v d u \\
\quad\left(C_{p}+i C_{s}\right) \\
\quad \int_{j=1}^{i} \int_{(j-1) t}^{j t} \int_{\delta((i-1) t-u)}^{i t-u}(1-r)^{i-j} \\
\cdot f_{U}(u) f_{V}(v) \\
\left.\cdot\left(1-F_{H}(i t-u-v)\right) d v d u\right) .
\end{gathered}
$$

4.1.2. Expected Renewal Cycle Length $E L_{11}(t)$. The relevant length of each event multiplies by the renewal probability, 


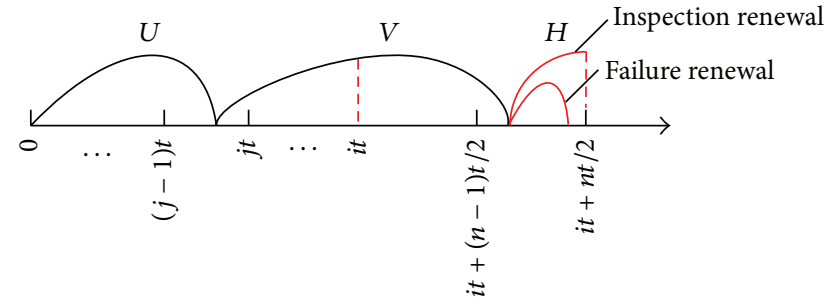

FIgURE 5: The system is renewed after minor defective stage is identified at the first time with imperfect inspection.

respectively; thus the expected renewal cycle length $E L_{11}(t)$ can be derived as

$$
\begin{gathered}
E L_{11}(t) \\
=\sum_{i=1}^{\infty}\left(\int_{0}^{t}((i-1) t+h)\right. \\
\cdot P\left((i-1) t+h<T_{f}<(i-1) t+h+d h\right) \\
\left.+i t P\left(T_{p}=i t\right)\right) \\
\quad \sum_{i=1}^{\infty}\left(\int_{0}^{t}((i-1) t+h)\right. \\
\quad+i t \sum_{j=1}^{i} \int_{(j-1) t}^{j t} \int_{\delta((i-1) t-u)}^{(i-1) t+h-u}(1-r)^{i-j} \\
\cdot f_{U}(u) f_{V}(v) f_{H} \\
\cdot((i-1-1) t-u) t+h-u-v) d v d u d h \\
\cdot f_{U}(u) f_{V}(v) \\
\cdot(1-r)^{i-j} \\
\left.\left.+F_{H}(i t-u-v)\right) d v d u\right) .
\end{gathered}
$$

4.2. The System Is Renewed after the Minor Defective Stage Is Identified at it, as Shown in Figure 5. There also exist two renewal scenarios.

(1) The system fails in $(i t+(n-1) t / 2$, it $+n t / 2)$ after the minor defective stage is identified at it at the first time. Inspections in $(j t,(i-1) t)$ all miss the minor defective stage. The minor defective stage ends within $(i t+(n-$ 1) $t / 2$, it $+n t / 2)$ and then severe defective stage and failure happens in the same interval. The probability of such a renewal is

$$
\begin{aligned}
& P\left(i t+\frac{(n-1) t}{2}<T_{f}<i t+\frac{n t}{2}\right) \\
& =P((j-1) t<U<j t, \\
& i t+\frac{(n-1) t}{2}-U<V<i t+\frac{n t}{2}-U, \\
& \left.0<H<i t+\frac{n t}{2}-U-V\right) \\
& =\sum_{j=1}^{i} \int_{(j-1) t}^{j t} \int_{i t+(n-1) t / 2-u}^{i t+n t / 2-u}(1-r)^{i-j} \\
& \cdot r f_{U}(u) f_{V}(v) F_{H}\left(i t+\frac{n t}{2}-u-v\right) d v d u .
\end{aligned}
$$

(2) The minor defective stage of system starts within $((j-$ 1) $t, j t)$ and is identified at it at the first time by an inspection. Then the subsequent inspection interval is halved. The length of severe defective stage is longer than $(i t+n t / 2-u-v)$ and is identified at $i t+n t / 2$. The probability of such a renewal is

$$
\begin{gathered}
P\left(T_{p}=i t+\frac{n t}{2}\right) \\
=P((j-1) t<U<j t, \\
i t+\frac{(n-1) t}{2}-U<V<i t+\frac{n t}{2}-U, \\
\left.H>i t+\frac{n t}{2}-U-V\right) \\
=\sum_{j=1}^{i} \int_{(j-1) t}^{j t} \int_{i t+(n-1) t / 2-u}^{i t+n t / 2-u}(1-r)^{i-j} \\
\cdot r f_{U}(u) f_{V}(v) \\
\cdot\left(1-F_{H}\left(i t+\frac{n t}{2}-u-v\right)\right) d v d u .
\end{gathered}
$$

Accordingly, the expected renewal cycle cost and length with different renewal scenarios can be derived as follows. 
4.2.1. Expected Renewal Cycle Cost $E C_{22}(t)$. The expected renewal cycle cost $E C_{22}(t)$ can be derived through multiplying the relevant cost of each event by the renewal probability; respectively;

$$
\begin{aligned}
& E C_{22}(t) \\
& =\sum_{i=1}^{\infty} \sum_{n=1}^{\infty}\left(\left(C_{f}+(i+n-1) C_{s}\right)\right. \\
& \cdot P\left(i t+\frac{(n-1) t}{2}<T_{f}<i t+\frac{n t}{2}\right) \\
& \left.+\left(C_{p}+(i+n) C_{s}\right) P\left(T_{p}=i t+\frac{n t}{2}\right)\right) \\
& =\sum_{i=1}^{\infty} \sum_{n=1}^{\infty}\left(\left(C_{f}+(i+n-1) C_{s}\right)\right. \\
& \cdot \sum_{j=1}^{i} \int_{(j-1) t}^{j t} \int_{i t+(n-1) t / 2-u}^{i t+n t / 2-u}(1-r)^{i-j} \\
& \cdot r f_{U}(u) f_{V}(v) F_{H} \\
& \cdot\left(i t+\frac{n t}{2}-u-v\right) d v d u \\
& +\left(C_{p}+(i+n) C_{s}\right) \\
& \cdot \sum_{j=1}^{i} \int_{(j-1) t}^{j t} \\
& \int_{i t+(n-1) t / 2-u}^{i t+n t / 2-u}(1-r)^{i-j} \\
& \cdot r f_{U}(u) f_{V}(v) \\
& \left.\cdot\left(1-F_{H}\left(i t+\frac{n t}{2}-u-v\right)\right) d v d u\right) \text {. }
\end{aligned}
$$

4.2.2. Expected Renewal Cycle Length $\mathrm{EL}_{22}(t)$. The relevant length of each event multiplies by the renewal probability, respectively; thus the expected renewal cycle length $E L_{22}(t)$ can be formulated as

$$
\begin{aligned}
& E L_{22}(t) \\
& =\sum_{i=1}^{\infty} \sum_{n=1}^{\infty} \int_{0}^{t / 2}\left(i t+\frac{(n-1) t}{2}+h\right) \\
& \cdot P\left(i t+\frac{(n-1) t}{2}+h<T_{f}<i t\right. \\
& \left.+\frac{(n-1) t}{2}+h+d h\right) \\
& +\left(i t+\frac{n t}{2}\right) P\left(T_{p}=i t+\frac{n t}{2}\right)
\end{aligned}
$$

$$
\begin{gathered}
=\sum_{i=1}^{\infty} \sum_{n=1}^{\infty}\left(\int_{0}^{t / 2}\left(i t+\frac{(n-1) t}{2}+h\right)\right. \\
\cdot \sum_{j=1}^{i} \int_{(j-1) t}^{j t}
\end{gathered}
$$$$
\begin{gathered}
\int_{i t+(n-1) t / 2-u}^{i t+(n-1) t / 2+h-u}(1-r)^{i-j} \\
\cdot r f_{U}(u) f_{V}(v) f_{H} \\
\cdot\left(i t+\frac{(n-1) t}{2}\right. \\
\quad+h-u-v) d v d u d h
\end{gathered}
$$$$
+\left(i t+\frac{n t}{2}\right)
$$$$
\sum_{j=1}^{i} \int_{(j-1) t}^{j t}
$$

$$
\begin{aligned}
& \cdot \int_{i t+(n-1) t / 2-u}^{i t+n t / 2-u}(1-r)^{i-j} \\
& \cdot r f_{U}(u) f_{V}(v) \\
& \cdot\left(1-F_{H}\right. \\
& \left.\left.\quad \cdot\left(i t+\frac{n t}{2}-u-v\right)\right) d v d u\right) .
\end{aligned}
$$

4.2.3. Expected Cost per Unit Time $C(t)$. Therefore, based on the derived expected cycle cost and length, expected cost per unit time can be calculated:

$$
C(t)=\frac{E C(t)}{E L(t)}=\frac{E C_{11}(t)+E C_{22}(t)}{E L_{11}(t)+E L_{22}(t)} .
$$

Model 2. It is shortening the inspection interval if only the minor defective stage is identified.

4.3. The System Is Renewed before Identifying the Minor Defective Stage with Imperfect Inspection. This scenario is the same as Section 4.1.

4.4. The System Is Renewed after the Minor Defective Stage Is Identified. As this model supposes that if only the minor defective stage is identified, the inspection interval is halved. The minor defective stage may be missed by the inspections because of the imperfect inspection. Moreover, the inspection may miss the minor defect between two points of identifying the minor defect. The modeling process is complex. Therefore, the scenario that system renews after identifying the minor defective stage at the third time is present as an example. The derivation of other scenarios follows the same principle. 


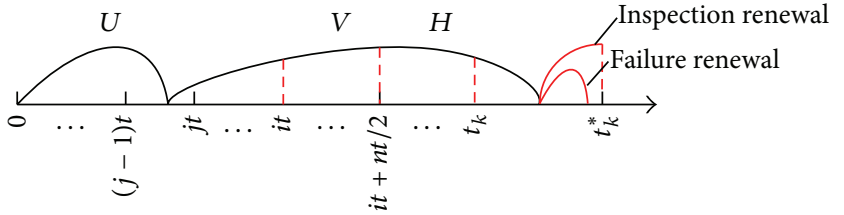

$t_{k}=i t+n t / 2+k t / 4$

$t_{k}^{*}=i t+n t / 2+k t / 4+t / 8$

Figure 6: The system is renewed after minor defective stage is identified at the third time with imperfect inspection.

The system is renewed after identifying the minor defective stage at the third time, as depicted in Figure 6.

(1) A failure renewal happens when the system deteriorates into the minor defective stage within $((j-$ $1) t, j t)$ and fails in $\left(t_{k}, t_{k}^{*}\right)$. The minor defective stage is identified three times in $\left(j t, t_{k}\right)$. The probability of such a failure renewal is

$$
\begin{gathered}
P\left(t_{k}<T_{f}<t_{k}^{*}\right) \\
=P\left((j-1) t<U<j t, t_{k}-U<V<t_{k}^{*}-U,\right. \\
\left.\quad 0<H<t_{k}^{*}-U-V\right) \\
=\sum_{j=1}^{i} \int_{(j-1) t}^{j t} \\
\cdot \int_{t_{k}-u}^{t_{k}^{*}-u}(1-r)^{(i-j+n+k-2)} r^{3} f_{U}(u) f_{V}(v) \\
\cdot F_{H}\left(t_{k}^{*}-u-v\right) d v d u .
\end{gathered}
$$

(2) An inspection renewal happens when the severe defective stage of system is identified at $t_{k}^{*}$. The minor defective stage lasts from $j t$ to $t_{k}$ and is identified by inspections three times. So the subsequent inspection interval is $t / 8$. The probability of this inspection renewal is

$$
\begin{gathered}
P\left(T_{p}=t_{k}^{*}\right) \\
=P\left((j-1) t<U<j t, t_{k}-U<V<t_{k}^{*}-U,\right. \\
\left.H>t_{k}^{*}-U-V\right) \\
=\sum_{j=1}^{i} \int_{(j-1) t}^{j t} \\
\cdot \int_{t_{k}-u}^{t_{k}^{*}-u}(1-r)^{(i-j+n+k-2)} r^{3} f_{U}(u) f_{V}(v) \\
\cdot\left(1-F_{H}\left(t_{k}^{*}-u-v\right)\right) d v d u .
\end{gathered}
$$

4.4.1. Expected Renewal Cycle Cost $E_{33}(t)$. The expected renewal cycle cost $E C_{33}(t)$ can be derived through multiplying the relevant cost of each event by the renewal probability, respectively;

$$
\begin{gathered}
E C_{33}(t) \\
=\sum_{i=1}^{\infty} \sum_{n=1}^{\infty}\left(\left(C_{f}+(i+n+k) C_{s}\right) P\left(t_{k}<T_{f}<t_{k}^{*}\right)\right. \\
\left.+\left(C_{p}+(i+n+k+1) C_{s}\right) P\left(T_{p}=t_{k}^{*}\right)\right) \\
=\sum_{i=1}^{\infty} \sum_{n=1}^{\infty}\left(\left(C_{f}+(i+n+k) C_{s}\right)\right. \\
\cdot \sum_{j=1}^{i} \int_{(j-1) t}^{j t} \\
\cdot \int_{t_{k}-u}^{t_{k}^{*}-u}(1-r)^{(i-j+n+k-2)} \\
\cdot r^{3} f_{U}(u) f_{V}(v) F_{H} \\
\cdot\left(t_{k}^{*}-u-v\right) d v d u \\
\left.+(i+n+k+1) C_{s}\right) \\
\cdot \sum_{j=1}^{i} \int_{(j-1) t}^{j t} \\
\cdot \int_{t_{k}-u}^{t_{k}^{*}-u}(1-r)^{(i-j+n+k-2)} \\
\cdot r^{3} f_{U}(u) f_{V}(v) \\
\left.\cdot\left(1-F_{H}\left(t_{k}^{*}-u-v\right)\right) d v d u\right) .
\end{gathered}
$$

4.4.2. Expected Renewal Cycle Length $E L_{33}(t)$. The relevant length of each event multiplies by the renewal probability, respectively; thus the expected renewal cycle length $E L_{33}(t)$ can be formulated as

$$
\begin{gathered}
E L_{33}(t) \\
=\sum_{i=1}^{\infty} \sum_{n=1}^{\infty} \int_{0}^{t / 8}\left(t_{k}+h\right) P \\
\cdot\left(t_{k}+h<T_{f}<t_{k}+h+d h\right) \\
+t_{k}^{*} P\left(T_{p}=t_{k}^{*}\right) \\
=\sum_{i=1}^{\infty} \sum_{n=1}^{\infty}\left(\int_{0}^{t / 8}\left(t_{k}+h\right)\right. \\
\cdot \sum_{j=1}^{i} \int_{(j-1) t}^{j t} \\
\cdot \int_{t_{k}-u}^{t_{k}+h-u}(1-r)^{(i-j+n+k-2)}
\end{gathered}
$$




$$
\begin{gathered}
\cdot r^{3} f_{U}(u) f_{V}(v) f_{H} \\
\cdot\left(t_{k}+h-u-v\right) d v d u d h \\
+t_{k}^{*} \sum_{j=1}^{i} \int_{(j-1) t}^{j t} \\
\cdot \int_{t_{k}-u}^{t_{k}^{*}-u}(1-r)^{(i-j+n+k-2)} \\
\cdot r^{3} f_{U}(u) f_{V}(v) \\
\left.\cdot\left(1-F_{H}\left(t_{k}^{*}-u-v\right)\right) d v d u\right) .
\end{gathered}
$$

4.4.3. Expected Cost per Unit Time $C(t)$. Based on the derived expected cycle cost and length, expected cost per unit time can be formulated:

$$
C(t)=\frac{E C(t)}{E L(t)}=\frac{E C_{11}(t)+E C_{33}(t)}{E L_{11}(t)+E L_{33}(t)} .
$$

\section{Numerical Example}

A numerical example is presented in this section to compare the superiority of two models according to the different measures when identifying the minor defective stage, thus presenting a scientific maintenance decision for safety assessment of NPPs. In order to avoid the influence of relevant parameters, two sets of parameters are set up here corresponding with the perfect inspection and imperfect inspection, respectively. These three stages of failure process are assumed to follow Weibull distribution because of the perfect performance of Weibull distribution in describing the lifetime of system, as shown in

$$
f_{X_{n}}(x)=a_{n} b_{n}\left(a_{n} x\right)^{b_{n}-1} e^{-\left(a_{n} x\right)^{b_{n}}},
$$

where $a_{n}, b_{n}$ are scale parameter and shape parameter, respectively.

Example 1 (perfect inspection). As the total lifetime experiment of systems or equipment following to three-stage failure process is hard to implement, we cannot possess the complete experimental data at present. So the distribution and cost parameters are assumed in Table 1.

According to the given parameters and cost models which have been built in Sections 3 and 4, we can derive the outputs of proposed models and determine the optimal inspection interval through MATLAB programming. The results of models 1 and 2 with perfect inspection are shown in Figures 7 and 8 , respectively.

In order to make comparison for the two different measures when identifying the minor defective stage, the results of two models are put together to make it more clear and intuitive, as shown in Figure 9. We can see that the expected cost per unit time of model 1 is smaller than model 2 no matter
TABLE 1: The distribution and cost parameters.

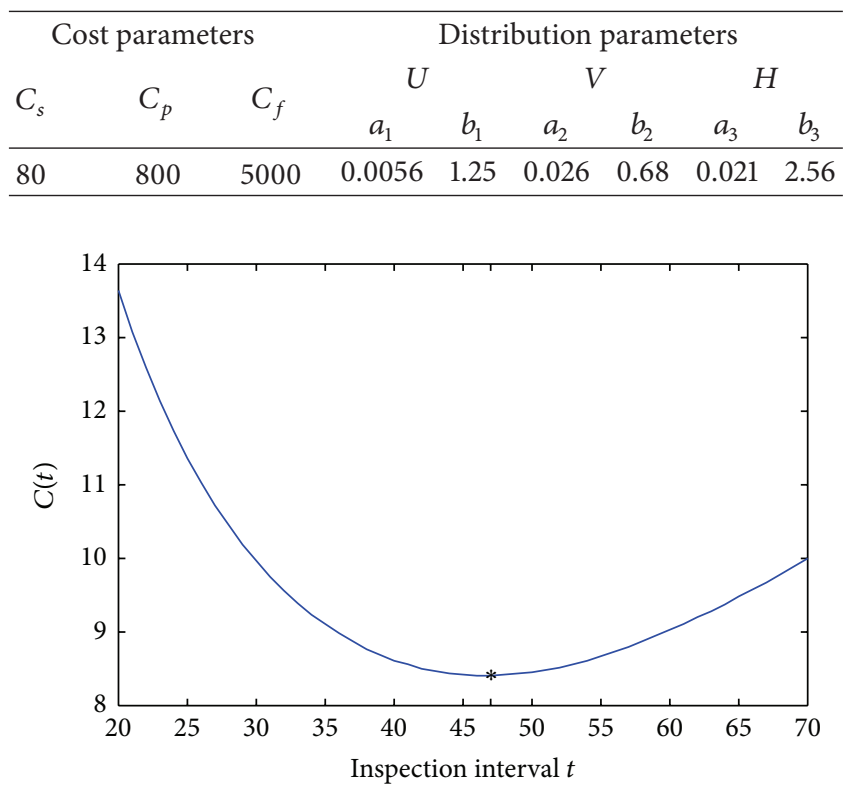

FIGURE 7: The result of model 1 with perfect inspection.

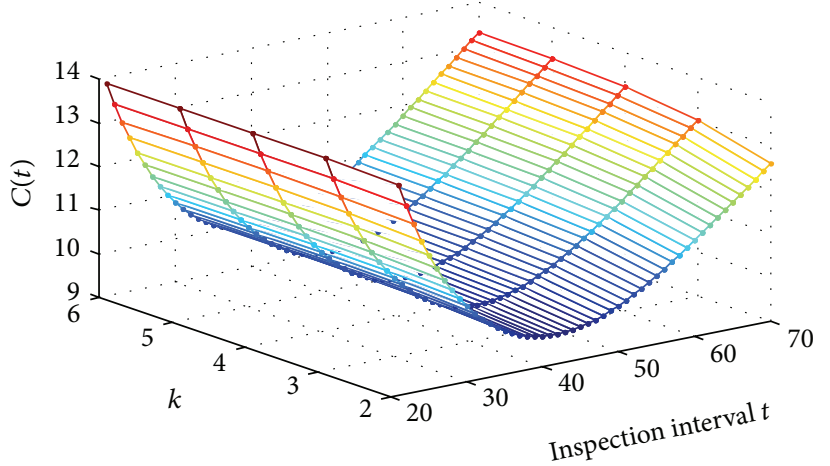

FIgURE 8: The result of model 2 with perfect inspection.

TABLE 2: The optimal results of models 1 and 2 with perfect inspection.

\begin{tabular}{llccc}
\hline$k$ & \multicolumn{2}{c}{ Model 2 } & \multicolumn{2}{c}{ Model 1 } \\
& $t^{*}$ & $C(t)$ & $t^{*}$ & $C(t)$ \\
\hline 2 & 42 & 9.6727 & & \\
3 & 41 & 9.8194 & & \\
4 & 41 & 9.8781 & 47 & $\mathbf{8 . 4 0 0 7}$ \\
5 & 41 & 9.9010 & & \\
6 & 41 & 9.9093 & & \\
7 & 41 & 9.9118 & & \\
\hline
\end{tabular}

what the value of $k$ is. Moreover, with the increasing of $k$ in model 2, the maintenance cost is larger. The relevant expected cost values of models 1 and 2 are given in Table 2 .

From Table 2, it can be seen that the optimal expected cost per unit time $C(t)$ of model 1 is 8.4007 and the optimal inspection interval $t^{*}$ is 47 . However, the optimal $C(t)$ is 9.6727 when the optimal $k^{*}$ is 2 and optimal $t^{*}$ is 42 . Therefore, model 1 can 


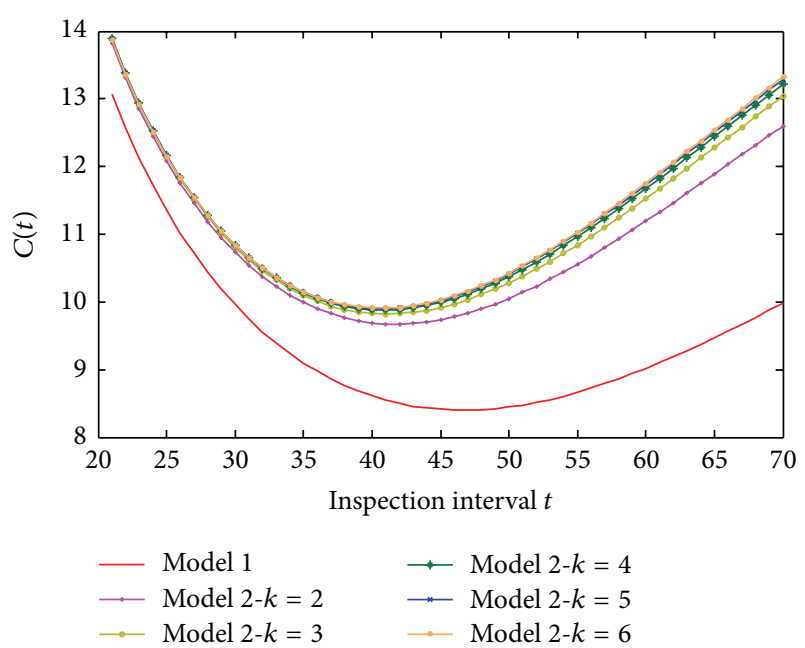

FIGURE 9: The results of two models with perfect inspection.

TABLE 3: The distribution and cost parameters.

\begin{tabular}{cccccccccc}
\hline & \multicolumn{4}{c}{ Cost parameters } & \multicolumn{8}{c}{ Distribution parameters } \\
$C_{s}$ & $C_{p}$ & $C_{f}$ & $a_{1}$ & $b_{1}$ & $a_{2}$ & $b_{2}$ & $a_{3}$ & $b_{3}$ \\
\hline 50 & 200 & 1500 & 0.023 & 1.15 & 0.058 & 1.47 & 0.16 & 1.14 \\
\hline
\end{tabular}

TABLE 4: The optimal results of models 1 and 2 with imperfect inspection.

\begin{tabular}{lcccr}
\hline & \multicolumn{2}{c}{ Model 2 } & \multicolumn{2}{c}{ Model 1 } \\
& $t^{*}$ & $C(t)$ & $t^{*}$ & $C(t)$ \\
\hline Case 1 & 16 & 20.4592 & & \\
Case 2 & 16 & 20.1346 & $\mathbf{1 3}$ & $\mathbf{1 7 . 9 2 1 6}$ \\
Case 3 & 15 & 20.3175 & & \\
\hline
\end{tabular}

save cost $13.15 \%$ more than model 2 . So model 1 is optimal compared with model 2 conditioning on the cost parameters. In other words, the measure that shortening the inspection interval only when identifying the minor defective stage at the first time can save more maintenance cost. Although the action of model 2 can prevent the failure of system definitely, frequent inspection maintenance will increase the maintenance cost. Therefore, the optimal maintenance decision is the action that shortening the inspection interval only when the minor defective stage is identified at the first time, which is also beneficial for making up the maintenance plan and improving the reliability and safety of NPPs.

Example 2 (imperfect inspection). In this section, we give another set of parameters to avoid the influence of different parameters. The distribution and cost parameters are shown in Table 3. The probability of imperfect inspection $r$ is 0.6 .

According to the given distribution and cost parameters, the outputs of models 1 and 2 are shown in Figure 10. The relevant expected cost values of models 1 and 2 are given in Table 4. With imperfect inspection case, model 1 can save maintenance cost $12.4 \%$ more than model 2 with condition 1 .

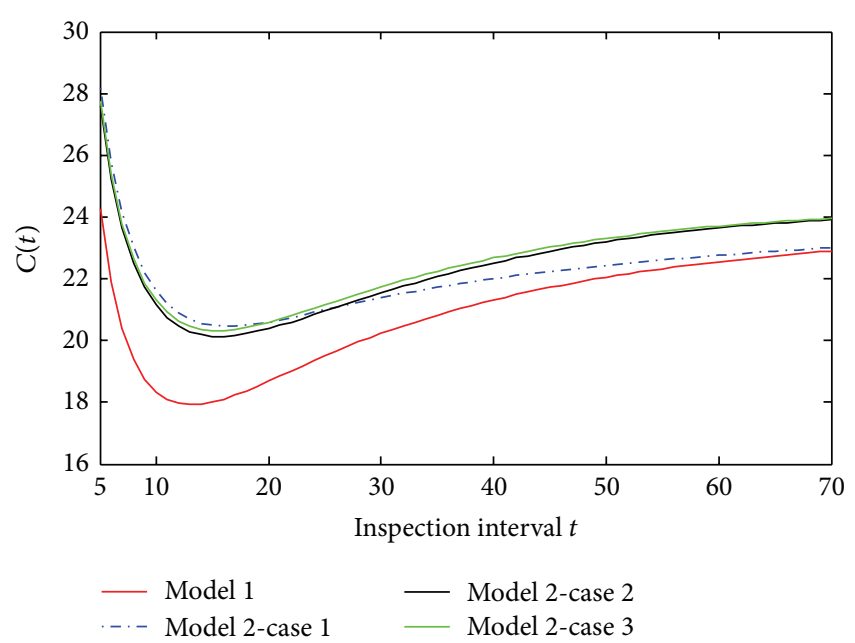

FIGURE 10: The results of two models with imperfect inspection.

From Figure 10 and Table 4, we can derive the same conclusion as Example 1 that model 1 is more optimal than model 2.

According to the two examples in this section, we can derive the conclusion that shortening the inspection interval only when the minor defective stage is identified at the first time is optimal no matter the inspection is perfect or imperfect. Moreover, this conclusion can help maintenance managers make scientific decisions, which will also be helpful for safety assessment of NPPs. Of course, the results cannot be generated to all cases as the output result of proposed models depends on the model parameters, particularly the cost parameters.

\section{Conclusions}

As evaluating the system reliability is one of the important tasks of PSA for NPPs and maintenance is considered as one of the most important factors for affecting the total reliability and safety, an enhanced preventive maintenance optimization model based on a three-stage failure process with perfect and imperfect inspection cases is proposed to optimize the inspection interval of NPPs by minimizing the expected cost per unit time. The lifetime of system before failure is divided into three stages, namely, normal, minor defective, and severe defective stages, which is closer to reality and corresponds to the three-color scheme commonly used in industry. When the minor defective stage is identified, two measures are considered for comparison: one is that halving the inspection interval only when the minor defective stage is identified at the first time; the other one is that if only identifying the minor defective stage, the subsequent inspection interval is halved. Maintenance is implemented immediately once the severe defective stage is identified. The results of the numerical example show that the measure that halving the inspection interval when the minor defective stage is identified at the first time can save more maintenance cost no matter the inspection is perfect or imperfect. 
The optimal inspection interval can be found through the proposed model. The conclusions can also help the managers make scientific maintenance decisions and benefit the safety assessment of nuclear power plants.

The further relevant research can be developed as (1) joint optimization policy of inspection and spare parts based on the three-stage failure process, (2) considering the maintenance window at the defective stages, and (3) case studies that should be considered. These points will be researched in the future.

\section{Conflict of Interests}

The authors declare no conflict of interests.

\section{Acknowledgment}

The project was supported by the Natural Science Foundation of Hebei Province (E2015506012).

\section{References}

[1] E. Hollnagel, "Human reliability assessment in context," Nuclear Engineering and Technology, vol. 37, no. 2, pp. 159-166, 2005.

[2] G. Heo and J. Park, "Framework of quantifying human error effects in testing and maintenance," in Proceedings of the 6th American Nuclear Society International Topical Meeting on Nuclear Plant Instrumentation, Control, and Human-Machine Interface Technologies, pp. 2083-2092, Knoxville, Tenn, USA, April 2009.

[3] T. Hamidouche, A. Bousbia-Salah, E. K. Si-Ahmed, and F. D'Auria, "Overview of accident analysis in nuclear research reactors," Progress in Nuclear Energy, vol. 50, no. 1, pp. 7-14, 2008.

[4] T. V. Santosh, G. Vinod, R. K. Saraf, A. K. Ghosh, and H. S. Kushwaha, "Application of artificial neural networks to nuclear power plant transient diagnosis," Reliability Engineering and System Safety, vol. 92, no. 10, pp. 1468-1472, 2007.

[5] International Atomic Energy Agency, "Implementation strategies and tools for condition based maintenance at nuclear power plants," Tech. Rep. IAEA-TECDOC-1551, IAEA, Vienna, Austria, 2007.

[6] E. K. Doyle, "On the application of stochastic models in nuclear power plant maintenance," European Journal of Operational Research, vol. 154, no. 3, pp. 673-690, 2004.

[7] S. Turki, Z. Hajej, and N. Rezg, "Impact of delivery time on optimal production/delivery/maintenance plans," in Proceedings of the 8th IEEE International Conference on Automation Science and Engineering (CASE '12), pp. 335-340, Seoul, Republic of Korea, August 2012.

[8] A. N. Das and S. P. Sarmah, "Preventive replacement models: an overview and their application in process industries," European Journal of Industrial Engineering, vol. 4, no. 3, pp. 280-307, 2010.

[9] H. Z. Wang, "A survey of maintenance policies of deteriorating systems," European Journal of Operational Research, vol. 139, no. 3, pp. 469-489, 2002.

[10] J.-M. Zhao and J.-D. Bai, "Optimal functional inspection policy with considering delay of repair," Systems Engineering and Electronics, vol. 31, no. 5, pp. 1255-1258, 2009.
[11] M. Bartholomew-Biggs, B. Christianson, and M. J. Zuo, "Optimizing preventive maintenance models," Computational Optimization and Applications, vol. 35, no. 2, pp. 261-279, 2006.

[12] R. P. Nicolai and R. Dekker, "Optimal maintenance of multicomponent systems: a review," in Complex System Maintenance Handbook, D. N. P. Murthy and A. K. S. Kobbacy, Eds., Springer, Amsterdam, The Netherlands, 2008.

[13] W. P. Pierskalla and J. A. Voelker, "A survey of maintenance models: the control and surveillance of deteriorating systems," Naval Research Logistics Quarterly, vol. 23, no. 3, pp. 353-388, 1976.

[14] J. Cai, H. F. Zuo, and H. W. Wang, "Optimal inspection model using delay-time concept," Journal of Applied Sciences, vol. 25, no. 4, pp. 26-31, 2007.

[15] J. Zhao and X. Jia, "An optimal policy of inspection for a component with delayed repair," Journal of Maintenance and Reliability, vol. 43, no. 3, pp. 20-23, 2009.

[16] A. H. Christer, "Innovative decision making," in Proceedings of the NATO Conference on the Role and Effectiveness of Theories of Decision in Practice, pp. 368-377, Hodder \& Stoughton, 1976.

[17] W. Y. Lu and R. Zheng, "The delay-time-based inspection models and their comparison," Journal of Management Sciences, vol. 20, no. 1, pp. 18-21, 2007.

[18] W. Wang, P. A. Scarf, and M. A. J. Smith, "On the application of a model of condition-based maintenance," Journal of the Operational Research Society, vol. 51, no. 11, pp. 1218-1227, 2000.

[19] W. Wang, "An inspection model based on a three-stage failure process," Reliability Engineering \& System Safety, vol. 96, no. 7, pp. 838-848, 2011.

[20] W. Wang, F. Zhao, and R. Peng, "A preventive maintenance model with a two-level inspection policy based on a three-stage failure process," Reliability Engineering and System Safety, vol. 121, pp. 207-220, 2014.

[21] R. F. Yang, F. Zhao, J. S. Kang, H. P. Li, and H. Z. Teng, "Inspection optimization model with imperfect maintenance based on a three-stage failure process," Journal of Maintenance and Reliability, vol. 17, no. 2, pp. 165-173, 2015.

[22] R. F. Yang, Z. W. Yan, and J. S. Kang, "An inspection maintenance model based on a three-stage failure process with imperfect maintenance via Monte Carlo simulation," International Journal of System Assurance Engineering and Management, 2014.

[23] R. F. Yang, F. Zhao, J. S. Kang, and X. H. Zhang, "An inspection optimization model based on a three-stage failure process," International Journal of Performability Engineering, vol. 10, no. 7, pp. 775-779, 2014.

[24] S. M. Ross, Introduction to Probability Models, Elsevier, New York, NY, USA, 2007. 


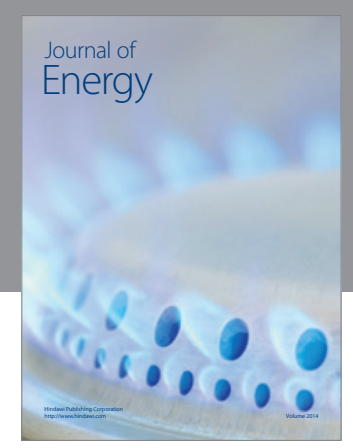

Journal of

Industrial Engineering
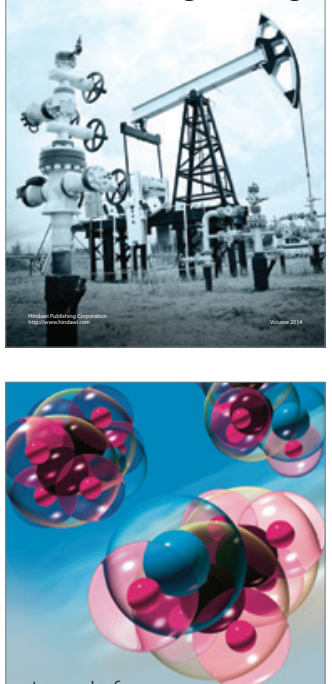

Fuels
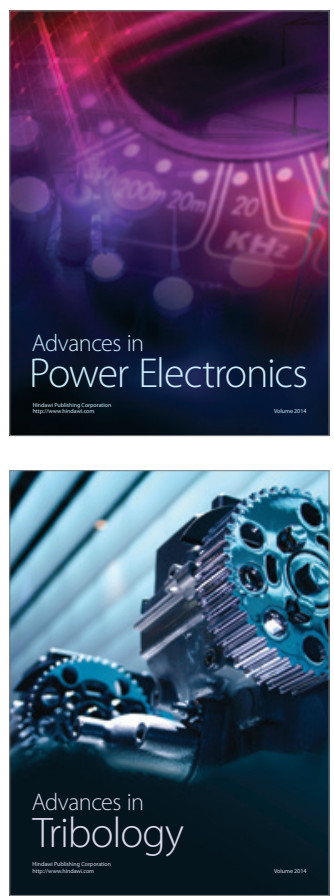

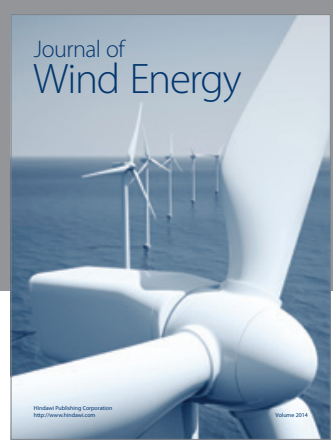

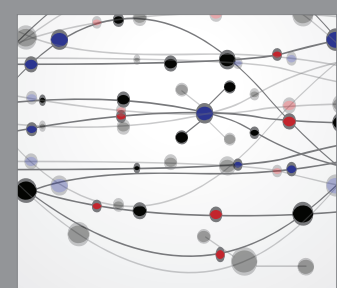

The Scientific World Journal

Submit your manuscripts at http://www.hindawi.com

Journal of

Structures
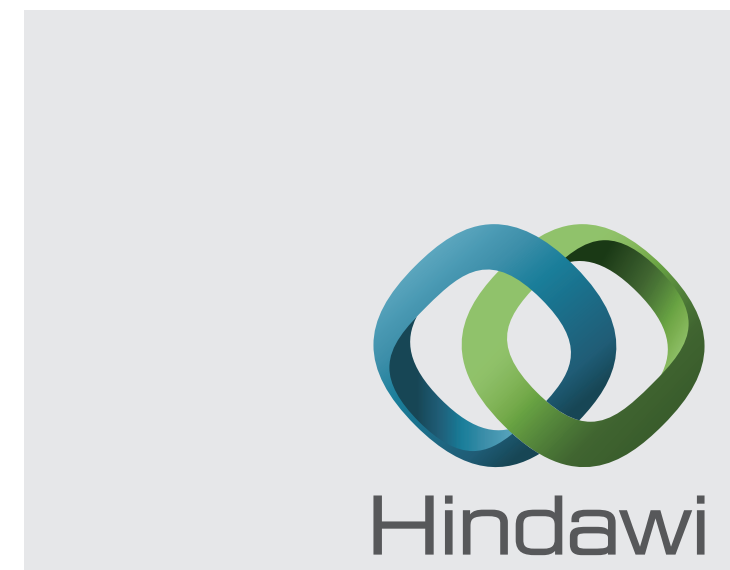

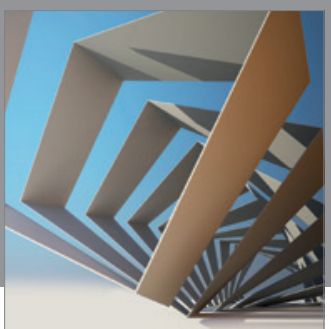

Rotating

Machinery
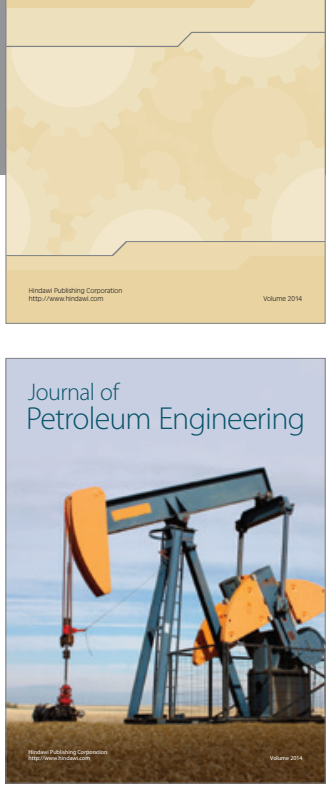

Journal of

Solar Energy
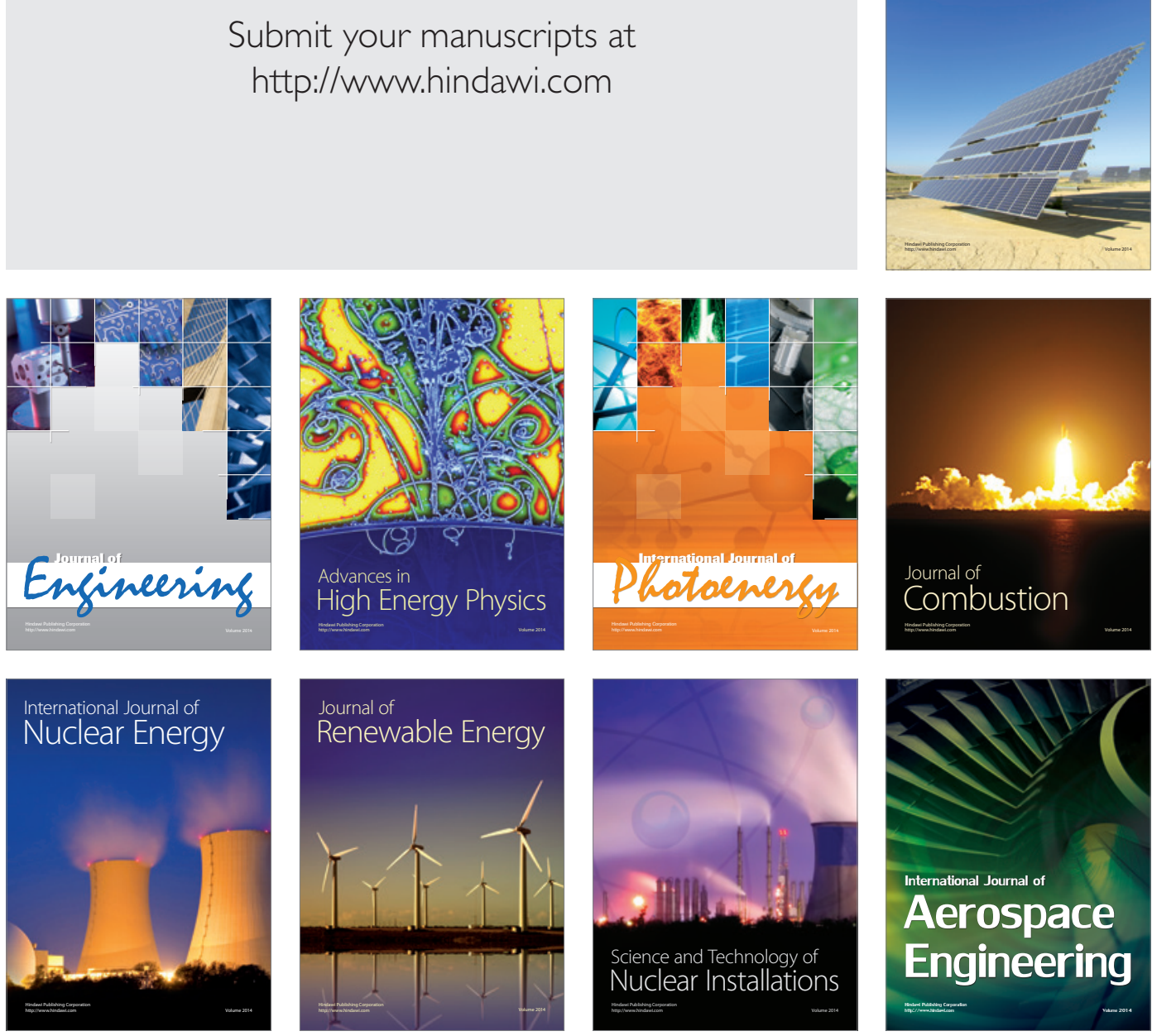